\title{
Breastmilk Output in a Disadvantaged Community with High HIV Prevalence as Determined by the Deuterium Oxide Dose-to-Mother Technique
}

\author{
Helen Mulol and Anna Coutsoudis
}

\begin{abstract}
Introduction: World Health Organization breastfeeding guidelines for HIV-infected mothers are exclusive breastfeeding for 6 months and then continued breastfeeding for 12 months, provided the mother is receiving antiretroviral prophylaxis. Many African women perceive that breastmilk alone is not sufficient for their infant's nutritional requirements for the first 6 months of life, and mixed feeding is a common practice.

Methodology: A stable isotope technique was used to determine breastmilk output volumes and maternal body composition objectively at five different time points in the first year of the infant's life.

Results: Breastmilk output volumes were high for HIV-infected mothers: $831 \pm 185 \mathrm{~g} / \mathrm{day}$ at 6 weeks; $899 \pm 188 \mathrm{~g} /$ day at 3 months; $871 \pm 293 \mathrm{~g} /$ day at 6 months; $679 \pm 281 \mathrm{~g} /$ day at 9 months; and $755 \pm 287 \mathrm{~g} /$ day at 12 months. These high output volumes had no negative impact on the mother's fat-free mass. The breastmilk output volumes for HIV-uninfected mothers were not significantly different to the outputs for HIV-infected mothers at any of the time points $(p>0.05)$ : $948 \pm 223 \mathrm{~g} /$ day at 6 weeks; $925 \pm 227 \mathrm{~g} /$ day at 3 months; $902 \pm 286 \mathrm{~g} /$ day at 6 months; $746 \pm 263 \mathrm{~g} /$ day at 9 months; and $713 \pm 264 \mathrm{~g} /$ day at 12 months.

Conclusion: This study using objective methodology shows that breastmilk outputs of HIV-infected mothers were relatively high (and within published reference ranges), and mothers are able to provide sufficient breastmilk for their infants without compromising their own fat-free mass.
\end{abstract}

\section{Introduction}

T he World Health Organization (WHO) recommends HIV-infected women should breastfeed exclusively for 6 months and then continue breastfeeding for 12 months or longer, provided that mothers receive antiretroviral (ARV) prophylaxis during the breastfeeding period. ${ }^{1}$ The use of ARVs has enabled mothers to breastfeed with less fear of transmitting the HIV virus to their infants, although this is a challenge in a setting where the extended family are often caregivers for the infant and HIV status is frequently not disclosed, not even to family and friends. Previously, HIV-infected mothers in South Africa were encouraged to formula feed to prevent transmission of HIV through the breastmilk if they satisfied the criteria for safe formula feeding. ${ }^{2}$ However, clinics ran out of formula, which often led to mothers diluting the formula or using other liquids to feed their infants with subsequent risks to infant health. In the light of evidence that exclusive breastfeeding was beneficial to infant health, even in the light of the chal- lenges of HIV, South African infant feeding policies underwent dramatic changes in 2011 with resultant repositioning, protection, promotion, and support of breastfeeding. ${ }^{3}$ These rapidly changing messages appeared to be conflicting and left some mothers, health workers, and the community confused about safe infant feeding practices.

Mixed feeding is common practice in South Africa, even where infants start with exclusive breastfeeding ${ }^{4}$ (or exclusive formula feeding). The risks associated with exclusive breastfeeding compared with mixed feeding with formula and solid foods have been reported. ${ }^{5,6}$ Infants who were exclusively breastfed were less likely to become infected with HIV than the latter, and mortality was reduced.

A gold-standard stable isotope method has enabled objective determination of the infant's breastmilk intake and, by inference, the breastmilk output of the mother. ${ }^{7,8}$ The method also simultaneously yields information about the mother's body composition. Breastmilk intake volumes determined from the stable isotope technique were found to be comparable to the test weighing method. ${ }^{9}$ This latter method is

Department of Pediatrics and Child Health, University of KwaZulu-Natal, Durban, South Africa. 
thought to underestimate the intake of breastmilk due to insensible water losses during feeding; it disrupts the normal pattern of feeding and is therefore inconvenient for the mother, especially when she is feeding at night and on demand, and these feeds may be excluded. ${ }^{10}$

Maternal body composition during lactation is of interest in a population that is poor and especially if the mother is HIV-infected, since maternal weight loss and loss of fat-free mass in HIV-infected mothers can put an increased burden on the mother's nutritional status. ${ }^{11}$ Hence, this study also investigated maternal body composition according to the maternal HIV status. Many African studies have focused only on weight changes during lactation, for example in Rwanda, ${ }^{12}$ Zambia, ${ }^{13}$ and a study of five African sites, including one in Durban, South Africa. ${ }^{14}$ A review of the published studies of body composition of lactating women found that the results were very variable, showing both increases and decreases in maternal weight during lactation. ${ }^{15}$ Some studies have used maternal weight and BMI as indicators, and studies related to breastfeeding have yielded mixed results and followed up mothers for different periods of time. ${ }^{16,17} \mathrm{~A}$ variety of methods are available to determine maternal body composition. Some studies have assessed maternal body fat by skinfold thickness, ${ }^{18,19}$ but these are reported to be subject to errors in measurement. ${ }^{20}$ Underwater weighing ${ }^{21}$ and the $B o d$ $P o d^{17}$ can also be used to determine body density and body fat. In a study in South Africa, maternal body composition during lactation was measured by bioimpedance spectrometry from 8 to 24 weeks post-delivery. ${ }^{22}$

The deuterium dilution method can also be used to determine maternal body composition in terms of whole-body fat and fat-free mass and was used in a study looking at whether nutritional supplementation would alter fat-free body mass in lactating mothers, which is of particular importance for HIV-infected mothers. ${ }^{23}$ To the authors' knowledge, there are few studies that have measured postdelivery maternal fat-free mass changes in conjunction with determination of the breastmilk output volumes, using the deuterium dilution technique for both measurements. Two studies of lactating mothers were carried out in different communities in Kenya, but both studies had limited sample sizes of only 10 mothers. ${ }^{24,25}$ A study in Mexico used the deuterium dilution technique to measure maternal body composition, but breastmilk intake volumes were determined by the test weighing technique. ${ }^{26}$ Neither of these studies looked at the effect of lactation on maternal body composition in the context of HIV-infected mothers. Therefore, this study will add important information to the field.

\section{Materials and Methods}

\section{Study population}

The study site was the Umkhumbane Community Health Centre (also known as the Cato Manor Clinic) in Cato Manor, Durban, South Africa, which is situated approximately $8 \mathrm{~km}$ from the city center. The area is characterized by high unemployment and high HIV prevalence rates, and contains a mixture of both formal and informal dwellings, many of which do not have access to a piped water supply and flush toilets in their homes. Infants in this disadvantaged community are therefore at risk when using mixed or replacement feeding.
Mothers were recruited from antenatal or postnatal visits at the Cato Manor Clinic to participate in the Improved Nutrition Program, which took place concurrently at the clinic and was conducted by the Department of Paediatrics and Child Health of the University of KwaZulu-Natal. The program aimed to educate, support, and encourage mothers to breastfeed their infants regardless of maternal HIV status, and covered topics such as correct positioning of the infant, common breastfeeding problems, introduction of complementary foods, gardening, and general life skills. Mothers who participated in the Cato Manor Clinic Improved Nutrition Program with a new baby and met the study's inclusion and exclusion criteria were invited to take part in this study (The Breast Milk Intake Study), which involved five visits at the following time points post-delivery: 6 weeks, and 3, 6, 9, and 12 months. After being informed about the study requirements and procedures, all mothers signed a consent to participate. Recruitment continued until 100 mother-infant pairs were enrolled and had completed at least one study time point. Ethical approval (BE211/11) was obtained from the University of KwaZulu-Natal Biomedical Research Ethics Committee (UKZN-BREC).

Inclusion criteria for the mother were initially that the mother was healthy, had no HIV or other infectious disease, intended to breastfeed her baby for 12 months, was participating in the Improved Nutrition Program, intended to live in the neighborhood of the clinic for 12 months after delivery, and was African. However, the number of women recruited with these criteria was low. Hence, an amendment was approved by the UKZN-BREC to allow asymptomatic HIVinfected mothers to participate in the study from March 2013. Exclusion criteria for the mother were pregnancy and a body mass index (BMI) $<18.5 \mathrm{~kg} / \mathrm{m}^{2}$. Inclusion criteria for the infant were full term, and a birth weight $>2.3 \mathrm{~kg}$. Exclusion criteria for the infant were twins, any defect that interferes with feeding, and chronic illness (e.g., congenital heart disease, cerebral palsy, etc.).

Maternal HIV status was determined from the clinic records (infant's Road to Health card), but 2/100 mothers in the study did not want to disclose their HIV status. Of the resulting 98 mothers, 40 were HIV-infected. The mean BMI was $27.2 \pm 5.1 \mathrm{~kg} / \mathrm{m}^{2}$ for the $40 \mathrm{HIV}$-infected mothers and $26.7 \pm 4.7 \mathrm{~kg} / \mathrm{m}^{2}$ for the $58 \mathrm{HIV}$-uninfected mothers at enrolment. The difference in means was not statistically significant.

\section{Methods}

Breastmilk output volumes were determined using the dose-to-mother deuterium dilution method, whereby the mother drinks a 30-g dose of deuterium oxide, which mixes rapidly with her body water. ${ }^{7}$ Saliva samples are taken from the mother and infant prior to the dose and over a period of 14 days thereafter. The deuterium enrichment in the mother's and infant's saliva is measured compared to their pre-dose saliva sample using a Fourier Transform Infrared Spectrometer (FTIR), and these values are then fitted to model curves using the Solver function in Microsoft Excel ${ }^{\circledR}$, which minimizes the sum of the squares of the differences of the FTIR and model values. Values for the breastmilk intake of the infant and, by inference, the breastmilk output of the mother are then calculated from these data. ${ }^{7}$ 
Maternal body composition can be determined from the same deuterium dilution method used for measurement of breastmilk intake volumes. ${ }^{7}$ Since the mothers drink a dose of deuterium and the natural logarithm of the mother's deuterium enrichment when plotted against time gives a straight line, the intercept on the $y$-axis gives the deuterium enrichment at time 0 . The pool space or volume distribution of deuterium, $V_{\mathrm{D}}$, is calculated from the deuterium dose given to the mother divided by the deuterium enrichment at the intercept. This value is then adjusted, as some of the deuterium is also involved in non-aqueous isotopic exchange, mainly in proteins. This is estimated to amount to $4.1 \%$ of the pool space. Hence, the mother's total body water (TBW) is calculated as: TBW $(\mathrm{kg})=V_{\mathrm{D}} / 1.041$. Fat-free mass is then calculated using the mother's hydration coefficient, which is a constant $73.2 \%$ water in adults as follows:

Fat-free mass $(\mathrm{kg})=\mathrm{TBW}(\mathrm{kg}) / 0.732$.

Percentage fat-free mass can then be calculated using the mother's body weight.

The WHO 24-hour recall was used to determine mother's report of infant feeding, ${ }^{27}$ and infants were classified as exclusively breastfed if they received breastmilk, vitamins, mineral supplements, and medicine only, in line with the WHO definition of exclusive breastfeeding. ${ }^{28}$

\section{Statistical analysis}

Sample size. The sample size had been set up to assess the breastmilk output of mothers accurately, and it was initially assumed that there would be only HIV-uninfected mothers. Since the change in study resulted in $40 \%$ HIV-infected mothers being recruited, it was realized that it would be possible to examine differences in breastmilk output in the two different groups of mothers. With the sample size, there was an $80 \%$ power to detect a $200 \mathrm{~g} /$ day difference in breastmilk output between HIV-infected and HIV-uninfected mothers, which was considered to be a meaningful difference.

\section{Data analysis}

Box and whisker plots were constructed using Microsoft Excel and the two-sample independent $t$ tests were calculated at the 95\% confidence level using Open Epi Software. Bivariate linear regression analyses were carried out using Stata v13 (StataCorp, College Station, TX).

\section{Results}

\section{Breastmilk output}

During the analysis of the deuterium results and computation of breastmilk intake volumes, 9/332 values measured were excluded, as the breastmilk, non-milk oral, or total water intake was $>1700 \mathrm{~g} /$ day. This value has been used as the exclusion criterion in other studies, ${ }^{29}$ and values $>1500$ g/day are to be investigated further in line with the guidelines for the method. ${ }^{30}$ One value for total water intake was very low (108 g/day at 12 months), and this value was therefore not included in the statistical analysis. In addition, 17 results did not fit the model, some of which were due to incomplete sample collection or sampling errors and could not be included in the results. The number of measurements that were excluded is not unusual for a method as complex as this, and similar levels are reported in other published studies. ${ }^{31}$ Figure 1 shows the breastmilk intake volumes for all infants over the period 6 weeks to 12 months. In bivariate linear regression, these breastmilk intake volumes were not associated with infant birth weight, infant sex, maternal age, maternal BMI, or maternal education at any of the five time points $(p>0.05)$.

Mean breastmilk intake volumes for HIV-infected and -uninfected mothers and corresponding infant weights are shown in Table 1. The means of the breastmilk intake volumes according to maternal HIV status at each time point were compared, and no difference was statistically significant. The HIV-infected mothers' reports of infant feeding gave $90.5 \%, 92.9 \%$, and $70.4 \%$ as exclusively breastfeeding at 6 weeks, 3 months, and 6 months, respectively; and the HIV-uninfected mothers' reports gave $83.3 \%, 86.0 \%$, and $54.5 \%$ as exclusively breastfeeding at 6 weeks, 3 months, and 6 months, respectively.

\section{Fat-free mass measurements}

During the analysis of the deuterium results and the computed fat-free mass values, $5 / 342$ values measured were not valid and were therefore not included in the statistical analysis. In addition, nine results did not fit the model, some of which were due to incomplete sample collection or sampling errors and could not be included in the results.

Table 2 shows the change in maternal fat-free mass between two adjacent time points and over the full observation

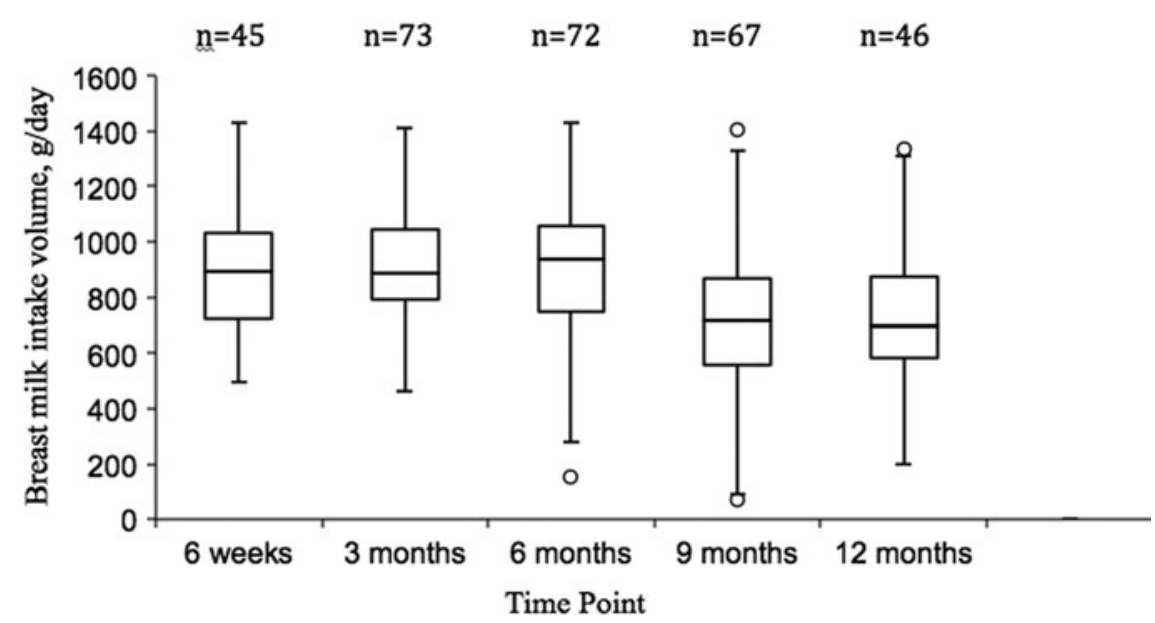

FIG. 1. Box and whisker plot of breastmilk intake volumes for all infants over the course of the study. 
Table 1. Mean Breastmilk Intake Volumes and Infant Weights According to Maternal HIV Status over the Course of the Study

\begin{tabular}{|c|c|c|c|c|c|}
\hline Time point & $\begin{array}{c}\text { BMIV } \\
\text { (HIV-infected } \\
\text { mother), g/day }\end{array}$ & $\begin{array}{c}\text { BMIV } \\
\text { (HIV-uninfected } \\
\text { mother), g/day }\end{array}$ & $\mathrm{p}-$ Value $e^{\mathrm{b}}$ & $\begin{array}{l}\text { Infant weight } \\
\text { (HIV-infected } \\
\text { mother), } \mathrm{kg}^{\mathrm{a}}\end{array}$ & $\begin{array}{l}\text { Infant weight } \\
\text { (HIV-uninfected } \\
\text { mother), } \mathrm{kg}^{\mathrm{a}}\end{array}$ \\
\hline 6 weeks & $831 \pm 185(n=21)$ & $948 \pm 223(n=24)$ & 0.06 & $4.84 \pm 0.57$ & $4.87 \pm 0.64$ \\
\hline 3 months & $899 \pm 188(n=28)$ & $925 \pm 227(n=45)$ & 0.61 & $6.11 \pm 0.72$ & $6.45 \pm 0.95$ \\
\hline 6 months & $871 \pm 293(n=27)$ & $902 \pm 286(n=45)$ & 0.66 & $7.35 \pm 0.87$ & $7.87 \pm 1.33$ \\
\hline 9 months & $679 \pm 281(n=24)$ & $746 \pm 263(n=43)$ & 0.33 & $8.36 \pm 1.15$ & $9.07 \pm 1.54$ \\
\hline 12 months & $755 \pm 287(n=13)$ & $713 \pm 264(n=33)$ & 0.64 & $9.31 \pm 0.80$ & $9.72 \pm 1.42$ \\
\hline
\end{tabular}

${ }^{\mathrm{a}}$ Mean \pm standard deviation.

${ }^{\mathrm{b}} t$ Test for BMIV.

BMIV, breastmilk intake volume.

period from 6 weeks $/ 3$ months to 12 months, according to the maternal HIV status. A two-sample independent $t$ test was used to compare the mean change in maternal fat-free mass, and there was no statistically significant difference in the means.

\section{Discussion}

The dose-to-mother deuterium dilution technique is considered by some to be a gold standard for measuring breastmilk output volumes. ${ }^{32}$ The breastmilk output volumes measured using this technique were high for both HIVinfected and -uninfected mothers for the first 6 months in comparison to the volumes reported in a pooled study of breastmilk intake in 12 countries using the same deuterium dilution technique. ${ }^{29}$ These pooled results gave mean breastmilk intake volumes of approximately $700 \mathrm{~g} /$ day at 1-2 months, $820 \mathrm{~g} /$ day at 3-4 months, and $810 \mathrm{~g} /$ day at 5-6 months, which were lower than those obtained in this study for HIV-infected and -uninfected mothers. The mean infant weights at the comparable time points of 3 and 6 months were lower than for the current study, which corroborates the higher breastmilk intake volumes seen in the present population, since other studies have reported a strong positive correlation between breastmilk intake volumes and infant weight. ${ }^{33}$ The 9-10-month intake in the pooled study was $>900 \mathrm{~g} /$ day $(n=75)$ but came from only three of the countries (Malawi, Papua New Guinea, and Zambia) and is higher than in the present study. The 11-12- and 12-24-month intake came from two countries (Gambia and Papua New Guinea; $n=53$ ) and was $800 \mathrm{~g} /$ day for $11-12$ months and $630 \mathrm{~g} /$ day for 12-24 months compared with the present study's mean of about 713-755 g/day at 12 months, depending on HIV status. These results are more difficult to interpret, since the effect of complementary feeding after 6 months is such as to make comparison between study populations more difficult. Furthermore, in the pooled study, the mean infant weights from 10 months were much lower than the mean infant weights at 8-9 months were, indicating that the results from the time points after 10 months were restricted to particular infant populations with much lower mean weights. ${ }^{29}$

A recent study also using the dose-to-mother deuterium dilution technique in Sri Lanka ${ }^{34}$ reported lower breastmilk intake volumes than the values obtained in the present study and, similar to the results from the pooled study, ${ }^{29}$ showed lower infant weights at comparable time points. For example, the 2- to <4-month breastmilk intake in Sri Lanka could be compared to the 6-week intake in South Africa if infant weights were taken into account rather than age. A longitudinal study of breastmilk intakes in exclusively breastfed infants using the test weighing method found a strong positive association between infant weight and the amount of breastmilk consumed by the infant. ${ }^{35}$ They compared their results with other studies and found a range of $600-900 \mathrm{~mL} /$ day breastmilk consumption during established lactation. A normal breastmilk intake is taken to be $150-200 \mathrm{~mL} / \mathrm{kg} / \mathrm{day}$ up to $5 \mathrm{~kg} .{ }^{36}$ Hence, for the mean infant weight of $4.86 \mathrm{~kg}$ at 6 weeks, normal total breastmilk intake should be 729 $972 \mathrm{~mL} /$ day. In the present study, the breastmilk intake volumes at 6 weeks for both HIV-infected and -uninfected mothers both fall in this range and can therefore be seen as adequate for infant feeding.

The changes in maternal fat-free mass were relatively small at all time points, and there were no significant

Table 2. Mean Change in Maternal Fat-Free Mass According to Maternal HIV Status over the Course of the Study

\begin{tabular}{lccc}
\hline Time points & $\begin{array}{c}\text { Change in FFM for } \\
\text { HIV-infected mother, } \mathrm{kg}^{\mathrm{a}}\end{array}$ & $\begin{array}{c}\text { Change in FFM for } \\
\text { HIV-uninfected mother, } \mathrm{kg}^{\mathrm{a}}\end{array}$ \\
\hline 6 weeks-3 months & $-0.6 \pm 3.4(n=15)$ & $+0.5 \pm 2.3(n=19)$ & 0.27 \\
3 months-6 months & $-0.2 \pm 3.1(n=24)$ & $+0.8 \pm 3.9(n=41)$ & 0.29 \\
6 months-9 months & $+0.4 \pm 2.8(n=23)$ & $-0.1 \pm 3.1(n=39)$ & 0.53 \\
9 months-12 months & $-0.2 \pm 3.4(n=20)$ & $0.0 \pm 2.1(n=34)$ & 0.84 \\
6 weeks-12 months & $-1.2 \pm 5.9(n=11)$ & $-0.2 \pm 2.9(n=32)$ & 0.53 \\
3 months-12 months & $-0.3 \pm 5.1(n=19)$ & 0.94 \\
\hline
\end{tabular}

${ }^{\mathrm{a}}$ Mean \pm standard deviation.

FFM, fat-free mass. 
differences in the means of HIV-infected compared to -uninfected mothers. In a poor pastoral community in Kenya where food insecurity was common and access to healthcare was limited, a study showed that BMI in lactating women decreased significantly, and maternal weight also decreased over the study period, although this did not reach significance. However, it is difficult to interpret these results due to the small sample size $(n=10)^{25}$ and the very different body composition compared with the current study - this Kenyan study reported a mean BMI that decreased from $19.8 \pm 1.3$ to $18.5 \pm 1.0 \mathrm{~kg} / \mathrm{m}^{2}$, whereas in the present study, mothers had a mean BMI at 6 weeks of $26.8 \pm 4.5 \mathrm{~kg} / \mathrm{m}^{2}$. In addition, the breastmilk output volumes in the Kenyan study were much lower $(552 \pm 22 \mathrm{~mL} /$ day $)$ than the volumes in the current study. In the present study, the mothers had a minimum BMI of $18.5 \mathrm{~kg} / \mathrm{m}^{2}$. Hence, it is possible that the results are not applicable to mothers with lower BMI values, even though there was no significant correlation in the present study between maternal BMI and the breastmilk output of the mother at BMI values $>18.5 \mathrm{~kg} / \mathrm{m}^{2}$. Furthermore, other studies have shown that there is no significant relationship between the mother's BMI and breastmilk output, even at BMI values $<18.5 \mathrm{~kg} / \mathrm{m}^{2} .37$

In a study in the same area in South Africa as this study, lactating mothers lost weight and fat-free mass over the study period of 2 weeks to 6 months, but there was not a significant difference in the amount of fat-free mass lost compared to the formula-feeding mothers. ${ }^{38} \mathrm{~A}$ study of the change in the maternal body composition over the period 2 weeks to 6 months in mothers who had/had not received nutritional supplementation $^{23}$ showed that mothers had no significant change in BMI, fat-free mass, and fat mass over the study period, which also indicates that the mothers in this area are not undernourished. Hence, the present findings confirm that even high breastmilk output volumes are not detrimental to a mother's health, as there was no significant loss of fat-free mass, and mothers can be encouraged to breastfeed regardless of HIV status.

\section{Limitations}

A limitation of the deuterium dilution method is that although it accurately assesses breastmilk intake over the period of observation, it may not represent breastfeeding practices outside this 14-day period. Breastfeeding patterns can vary according to the infant's needs and may not be constant over time. The study was powered to detect a difference in breastmilk output volume of $200 \mathrm{~g} / \mathrm{day}$, which was chosen to be a meaningful difference between HIV-infected and -uninfected mothers. This value may seem a rather large value, but in this study, anything less than this value would not be clinically significant, given that the mean breastmilk output volumes were relatively high in the first 6 months (>800 g/day).

\section{Conclusion}

This study using objective methodology shows that breastmilk outputs of HIV-infected mothers were relatively high (and within published reference ranges) and mothers are able to provide sufficient breastmilk for their infants without compromising their own fat-free mass. These data endorse the WHO and South African guidelines to encourage 12 months of breastfeeding for HIV-infected mothers.

\section{Acknowledgments}

The authors wish to acknowledge the assistance of Gcinile Maphanga, Zanele Msomi, Bathabile Zungu, and Nokukhanya Nzama in completing questionnaires and saliva sampling; Penny Reimers for breastfeeding counseling; Dr. Eshia Moodley for assistance with general study management; eThekwini Municipality for use of the study site; the mothers and infants who participated in this study; and Prof. Benn Sartorius for statistical analysis.

The study was funded by the International Atomic Energy Agency Research Contract No. 16844 and RAF 6039, University of KwaZulu-Natal College of Health Sciences, and the Medical Research Council.

\section{Disclosure Statement}

The authors declare no conflicts of interest.

\section{References}

1. World Health Organization. Consolidated guidelines on the use of antiretroviral drugs for treating and preventing HIV infection: recommendations for a public health approach. Geneva, Switzerland, 2013.

2. Doherty T, Chopra M, Nkonki L, et al. Effect of the HIV epidemic on infant feeding in South Africa: "When they see me coming with the tins they laugh at me." Bull World Health Organ 2006;84:90-96.

3. Department of Health. The Tshwane declaration of support for breastfeeding in South Africa. Tshwane, South Africa, 2011.

4. Doherty T, Chopra M, Nkonki L, et al. A longitudinal qualitative study of infant-feeding decision making and practices among HIV-positive women in South Africa. J Nutr 2006;136:2421-2426.

5. Coovadia HM, Rollins NC, Bland RM, et al. Mother-tochild transmission of HIV-1 infection during exclusive breastfeeding in the first 6 months of life: an intervention cohort study. Lancet 2007;369:1107-1116.

6. Coutsoudis A, Pillay K, Kuhn L, et al. Method of feeding and transmission of HIV-1 from mothers to children by 15 months of age: prospective cohort study from Durban, South Africa. AIDS 2001;15:379-387.

7. Coward WA. Measuring milk intake in breast-fed babies. J Pediatr Gastroenetrol Nutr 1984;3:275-279.

8. IAEA. Stable isotope technique to assess intake of human milk in breastfed infants. Human Health Series No. 7, Vienna. www-pub.iaea.org/MTCD/Publications/PDF/Pub1 429web.pdf (accessed March 24, 2015).

9. Butte NF, Wong WW, Patterson BW, et al. Human-milk intake measured by administration of deuterium oxide to the mother: a comparison with the test-weighing technique. Am J Clin Nutr 1988;47:815-821.

10. Butte NF, Wong WW, Klein PD, et al. Measurement of milk intake: tracer-to-infant deuterium dilution method. $\mathrm{Br}$ J Nutr 1991;65:3-14.

11. Knox TA, Zafonte-Sanders M, Fields-Gardner C, et al. Assessment of nutritional status, body composition, and human immunodeficiency virus-associated morphological changes. Clin Infect Dis 2003;36:S63-68.

12. Ladner J, Castetbon K, Leroy V, et al. Pregnancy, body weight and human immunodeficiency virus infection in African women: a prospective cohort study in Kigali (Rwanda), 1992-1994. Pregnancy and HIV Study Group (EGE). Int J Epidemiol 1998;27:1072-1077. 
13. Murnane PM, Arpadi SM, Sinkala M, et al. Lactationassociated postpartum weight changes among HIV-infected women in Zambia. Int J Epidemiol 2010;39:1299-1310.

14. Cames C, Cournil A, De Vincenzi I, et al. Postpartum weight change among HIV-infected mothers by antiretroviral prophylaxis and infant feeding modality in a research setting. AIDS 2014;28:85-94.

15. Butte NF, Hopkinson JM. Body composition changes during lactation are highly variable among women. $J$ Nutr 1998;128:381S-385S.

16. Gigante DP, Victora CG, Barros FC. Breast-feeding has a limited long-term effect on anthropometry and body composition of Brazilian mothers. J Nutr 2001;131:78-84.

17. Hatsu IE, McDougald DM, Anderson AK. Effect of infant feeding on maternal body composition. Int Breastfeed $J$ 2008;3:18.

18. Dewey KG, Heinig MJ, Nommsen LA. Maternal weightloss patterns during prolonged lactation. Am J Clin Nutr 1993;58:162-166.

19. Brewer MM, Bates MR, Vannoy LP. Postpartum changes in maternal weight and body fat depots in lactating vs nonlactating women. Am J Clin Nutr 1989;49:259-265.

20. Ulijaszek SJ, Kerr DA. Anthropometric measurement error and the assessment of nutritional status. Br J Nutr 1999;82: $165-177$.

21. AbuSabha R, Greene G. Body weight, body composition, and energy intake changes in breastfeeding mothers. J Hum Lact 1998;14:119-124.

22. Papathakis PC, Van Loan MD, Rollins NC, et al. Body composition changes during lactation in HIV-infected and HIV-uninfected South African women. J Acquir Immune Defic Syndr 2006;43:467-474.

23. Kindra G, Coutsoudis A, Esposito F. Effect of nutritional supplementation of breastfeeding HIV positive mothers on maternal and child health: findings from a randomized controlled clinical trial. BMC Public Health 2011;11:946.

24. Ettyang GA, Van Marken Lichtenbelt DW, Saris WHM, et al. Assessment of body composition in lactating mothers in a rural African community using deuterium oxide. SAJCN 2004;17:10-15.

25. Ettyang GA, Van Marken Lichtenbelt WD, Esamai F, et al. Assessment of body composition and breast milk volume in lactating mothers in pastoral communities in Pokot, Kenya, using deuterium oxide. Ann Nutr Metab 2005;49:110-117.

26. Barbosa L, Butte NF, Villalpando S, et al. Maternal energy balance and lactation performance of Mesoamerindians as a function of body mass index. Am J Clin Nutr 1997;66: $575-583$.

27. World Health Organisation. Indicators for assessing breastfeeding practices. Division of Child Health and Development. Geneva, Switzerland, 1991.
28. World Health Organisation. Indicators for assessing infant and young child feeding practices. Part 1 Definitions. Geneva, Switzerland, 2008.

29. Da Costa TH, Haisma H, Wells JC, et al. How much human milk do infants consume? Data from 12 countries using a standardized stable isotope methodology. J Nutr 2010; 140:2227-2232.

30. International Atomic Energy Agency. Guidance notes on evaluation of data in the Excel spreadsheet to calculate human milk intake by breastfed infants. https://nucleus.iaea.org/ HHW/Nutrition/MilkIntake/Draft_Guidance_notes_on_eval uation_of_data_in_the_Excel_spreadsheet_06_Feb_2015_ English_docx.pdf (accessed September 28, 2015).

31. Moore SE, Prentice AM, Coward WA, et al. Use of stableisotope techniques to validate infant feeding practices reported by Bangladeshi women receiving breastfeeding counseling. Am J Clin Nutr 2007;85:1075-1082.

32. Jarjou LMA, Goldberg GR, Coward WA, et al. Calcium intake of rural Gambian infants: a quantitative study of the relative contributions of breast milk and complementary foods at 3 and 12 months of age. Eur J Clin Nutr 2012;66: 673-677.

33. Dewey KG, Heinig MJ, Nommsen LA, et al. Maternal versus infant factors related to breast milk intake and residual milk volume: the DARLING study. Pediatrics 1991; 87:829-837.

34. Bandara T, Hettiarachchi M, Liyanage C, et al. The deuterium oxide-to-the-mother method documents adequate breast-milk intake among Sri Lankan infants. J Nutr 2015; 145:1325-1329.

35. Neville MC, Keller R, Seacat J, et al. Studies in human lactation: milk volumes in lactating women during the onset of lactation and full lactation. Am J Clin Nutr 1988; 48:1375-1386.

36. Riordan J. Breastfeeding and Human Lactation, 3rd ed. Sudbury, MA: Jones and Bartlett, 2004.

37. Prentice AM, Goldberg GR, Prentice A. Body mass index and lactation performance. Eur J Clin Nutr 1994;48:S78-86.

38. Kindra G, Coutsoudis A, Esposito F, et al. Breastfeeding in HIV exposed infants significantly improves child health: a prospective study. Matern Child Health J 2012;16:632-640.

Address correspondence to: Helen Mulol, MSc Department of Pediatrics and Child Health University of KwaZulu-Natal P/Bag 7, Congella 4013 South Africa

E-mail: Mulol@ukzn.ac.za 